\title{
CCD STRÖMGREN PHOTOMETRY OF YOUNG REDDENED CLUSTERS
}

\author{
Antonio J. Delgado, ${ }^{1}$ AND Emilio J. Alfaro ${ }^{1}$ \\ Instituto de Astrofísica de Andalucía, CSIC. P.O. Box 3004, E-18080, Granada, Spain \\ Electronic mail:delgado@iaa.es, emilio@iaa.es \\ JESÚS CABRERA-CAÑO \\ Facultad de Física, Universidad de Sevilla, P.O. Box 1045, E-41080, Sevilla, Spain \\ Electronic mail: jesus@obelix.cica.es \\ Received 1996 August 15; revised 1996 October 31
}

\begin{abstract}
The capabilities of CCD uvby photometry for the study of reddened young clusters are investigated. Observations of four fields in the direction of the clusters Basel 1, Berkeley 86, NGC 6704, and NGC 6756, with a total of 1665 stars measured in at least the $y$ and $b$ bands, produce new estimates for the clusters parameters. The results are compared to those from previously published studies in different photometric systems showing good agreement in the values of color excess and distance modulus, with the exception of NGC 6756, for which an essentially larger distance is found. Furthermore, the ages estimated for this cluster, Basel 1, and NGC 6704 are found to be higher than previously assumed, in particular for the last one. The presence of red giants as cluster members is suggested in Basel 1, NGC 6704, and NGC 6756. (C) 1997 American Astronomical Society. [S0004-6256(97)01702-0]
\end{abstract}

\section{INTRODUCTION}

A project on CCD uvby photometry of reddened young clusters has been running during the past three years, with the aims of gaining further insight in the peculiarities of reddened uvby colors for OB-type stars. Previous work (Manfroid \& Sterken 1987; Delgado \& Alfaro 1989; Alfaro \& Delgado 1991; Delgado et al. 1992) has revealed and discussed the existence of systematic errors in the intrinsic uvby colors, possibly dependent on spectral type, and/or color excess.

The $u v b y$ observation of young reddened clusters with a CCD offers some advantages in this context. With telescopes of moderate size, and reasonable integration times, it is possible to secure measurements for stars in a wide range of spectral types, from the most luminous OB-type cluster members, down to F-, and even early G-type main sequence stars. This allows a more reliable determination of the cluster parameters, and in particular, the analysis of the reddening affecting the cluster. When complemented with measurements in the $\mathrm{H} \beta$ system, individual determinations of the color excess are possible for the whole spectral type range covered, allowing in turn a measurement of the cluster metallicity. Finally, these observations help in refining the knowledge of the quantity and quality of the interstellar reddening in the direction of the cluster. In particular, the specific features of the extinction due to intracluster matter, as differenced from the diffuse interstellar extinction (Turner 1994, and references therein), can be investigated.

A selection of northern objects from the catalog of Lyngå,

${ }^{1}$ Visiting Astronomer, German-Spanish Astronomical Center, Calar Alto, operated by the Max Planck Institute für Astronomie, Heidelberg, jointly with the Spanish Comisión Nacional de Astronomía. with a color excess $\mathrm{E}(B-V)$ larger than 0.5 , and ages equal or less than $10^{8}$ years, provides a sample of about 50 clusters. The amount of published data for the clusters in this sample is inhomogeneous. Only a few have published measurements in the Strömgren system, and for many of them the catalogued values stem from old photometric studies in the $R G U$ photographic system. In this paper, we present the results of the first CCD observing campaigns, for the clusters Basel 1, Berkeley 86, NGC 6704, and NGC 6756. Strömgren photometry is usually complemented with measurements in the Crawford $\mathrm{H} \beta$-system. In fact, for stars of spectral type later than $\mathrm{A}$, the $\mathrm{H} \beta$ index is commonly used as the independent temperature parameter, and most calibrations, including those of metallicity, $[\mathrm{Fe} / \mathrm{H}]$, in terms of $\delta m_{1}$, make use of it. Additional work is under way, to complete the present data with measurements of the $\mathrm{H} \beta$ index, which, obviously enough, are more time consuming by an important factor, if one wants to achieve measurements with precision comparable to the one in $u v b y$ bands, for the stars in the lower main sequence.

In the next section we describe the reduction procedure and the transformation to the standard system. Section 3 contains the analysis of the photometric diagrams, calculation of the estimates for the different clusters parameters, and a comparison with results previously published. The last section resumes the main conclusions of our study.

\section{OBSERVATIONS AND DATA REDUCTION}

The observations were obtained at the $1.5 \mathrm{~m}$, and $1.23 \mathrm{~m}$ telescopes in the Calar Alto Observatory, in 1993 June. Figures $1(\mathrm{a})-1(\mathrm{~d})$, show plots of the observed fields, with the stars measured in at least $y$ and $b$ bands. The dot sizes are indicative of the relative instrumental $y$ magnitude in each

\section{(C) American Astronomical Society - Provided by the NASA Astrophysics Data System}



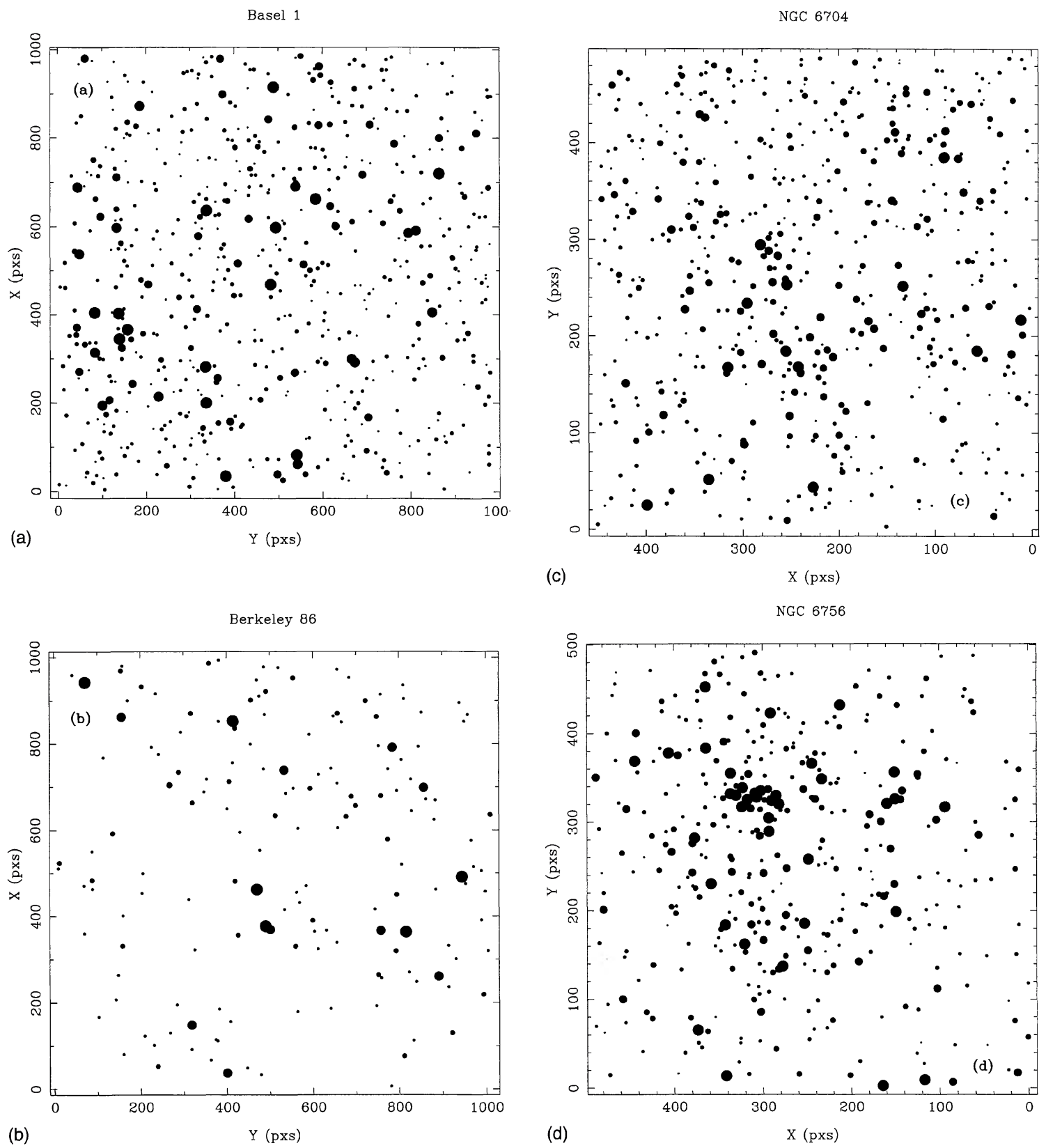

FIG. 1. (a)-(d). Schematic maps of the four cluster fields observed. Only stars measured in at least $y$ and $b$ are plotted. The size of the dots represents relative brightness of stars in the field. North is up and East left in all fields, with the exception of the field in Basel 1, where the North-South axis is clockwise rotated about 30 degrees.

frame. The dates, and detectors used in each telescope are, $1.5 \mathrm{~m}$ telescope: 1993 June 14-19, detector THX 1024 $\times 1024$, with $19 \mu / p x$, and $0.33^{\prime \prime} / p x .1 .23 \mathrm{~m}$ telescope: 1993 June $13-17$, detector Tex $420 \times 520$, with $27 \mu / p x$, and $0.57^{\prime \prime} / p x$. In Table 1 we include the schedule of the observations in both instruments, along with integration times, airmass at the middle of observation, and full width at half maximum in each frame.

The standard stars were selected from three sources, trying to cover the largest possible range in $(b-y)$ values. Primary standards were selected from the catalog by Olsen
(1983). Some stars were also taken from the measurements of B-type stars by Knude (1992) and from the compilation of Schuster \& Nissen (1988). However, the transformation to standard colors requires extrapolation of the calibration relations. In the absence of significantly reddened B-type standards, the effects of this extrapolation can be diminished by including late type stars in the standard sample. This can however introduce new uncertainties, since the variations of color indices due to reddening might differ from those due to differences in spectral type (Delgado \& Alfaro 1989). Table 
TABLE 1. Schedule of the observations.

\begin{tabular}{|c|c|c|c|c|}
\hline $\begin{array}{l}\text { Cluster } \\
\text { (date, Tl.) }\end{array}$ & Band & $\begin{array}{c}T_{i n t} \\
(\mathrm{~s})\end{array}$ & Airmass & $\begin{array}{c}\text { FWHM } \\
\quad(\mathrm{px})\end{array}$ \\
\hline Basel 1 & $y$ & 300 & 1.373 & 5.1 \\
\hline \multirow[t]{3}{*}{$16.6 .93,1.5$} & $\mathrm{~b}$ & 800 & 1.401 & 7.0 \\
\hline & $\mathrm{v}$ & 1200 & 1.441 & 5.7 \\
\hline & $\mathrm{u}$ & 3000 & 1.558 & 6.6 \\
\hline Berkeley 86 & $\mathrm{y}$ & 20 & 1.191 & 3.5 \\
\hline \multirow[t]{6}{*}{$17.6 .93,1.5$} & $y$ & 300 & 1.157 & 4.4 \\
\hline & $\mathrm{b}$ & 50 & 1.150 & 4.0 \\
\hline & $\mathrm{b}$ & 500 & 1.132 & 5.3 \\
\hline & $\mathrm{v}$ & 1200 & 1.083 & 5.3 \\
\hline & $\mathrm{v}$ & 120 & 1.062 & 3.8 \\
\hline & $\mathrm{u}$ & 3000 & 1.030 & 5.0 \\
\hline NGC 6704 & $\mathrm{y}$ & 620 & 1.356 & 3.2 \\
\hline \multirow[t]{3}{*}{$14.6 .93,1.23$} & $\mathrm{~b}$ & 1180 & 1.360 & 3.4 \\
\hline & $\mathrm{v}$ & 1500 & 1.383 & 4.4 \\
\hline & $\mathrm{u}$ & 3600 & 1.484 & 4.5 \\
\hline \multirow[t]{4}{*}{$15.6 .93,1.23$} & $y$ & 960 & 1.425 & 3.5 \\
\hline & $\mathrm{b}$ & 1360 & 1.361 & 3.5 \\
\hline & $\mathrm{v}$ & 1500 & 1.355 & 3.8 \\
\hline & $\mathrm{u}$ & 3600 & 1.400 & 4.0 \\
\hline NGC 6756 & $y$ & 1080 & 1.345 & 4.0 \\
\hline \multirow[t]{3}{*}{$16.6 .93,1.23$} & $\mathrm{~b}$ & 2000 & 1.226 & 4.9 \\
\hline & $\mathrm{v}$ & 3000 & 1.191 & 4.6 \\
\hline & $\mathrm{u}$ & 3600 & 1.209 & 5.5 \\
\hline
\end{tabular}

2 lists the standard stars measured in both telescopes, with number of measurements and references.

The reduction of all frames was done with the IRAF routines for the bias, dark and flat-field corrections, and the registering and combination of frames. The frames listed in Table 1 are those actually used for photometry. The integration times in this table are in some cases (Basel 1, $y$; NGC 6704 , NGC 6756 all bands) the result of adding several consecutive frames, after previous registering of all frames for

TABLE 2. Standard stars observed.

\begin{tabular}{ccccccccr}
\hline \hline & & & & & \multicolumn{5}{c}{$\mathrm{n}$} \\
$\mathrm{HD}$ & $V$ & $b-y$ & \multicolumn{1}{c}{$m_{1}$} & $c_{1}$ & $\mathrm{Sp}$ & 1.23 & 1.50 & Ref \\
\hline 133769 & 8.541 & 0.181 & 0.270 & 0.778 & $\mathrm{~F} 0$ & 6 & 2 & 1 \\
134248 & 8.372 & 0.402 & 0.205 & 0.343 & $\mathrm{G} 0$ & 8 & 5 & 1 \\
135200 & 8.122 & 0.271 & 0.143 & 0.616 & $\mathrm{~F} 0$ & 4 & 6 & 1 \\
135262 & 8.563 & 0.457 & 0.199 & 0.434 & $\mathrm{G} 0$ & 2 & 4 & 1 \\
161848 & 8.911 & 0.489 & 0.340 & 0.283 & $\mathrm{~K} 0$ & 3 & 0 & 3 \\
165503 & 7.844 & 0.160 & 0.220 & 0.749 & $\mathrm{~F} 0$ & 3 & 7 & 1 \\
166179 & 8.328 & 0.188 & 0.168 & 0.693 & $\mathrm{~F} 0$ & 1 & 0 & 1 \\
166600 & 8.639 & 0.277 & 0.185 & 0.748 & $\mathrm{G} 0$ & 5 & 4 & 1 \\
166710 & 8.353 & 0.497 & 0.246 & 0.428 & $\mathrm{G} 0$ & 2 & 3 & 1 \\
166928 & 8.532 & 0.255 & 0.121 & 1.100 & $\mathrm{~A} 5$ & 7 & 11 & 1 \\
164760 & 7.959 & 0.163 & 0.038 & 0.958 & $\mathrm{~B} 9$ & 3 & 3 & 1 \\
165343 & 8.490 & 0.151 & 0.048 & 0.648 & $\mathrm{~B} 8$ & 1 & 3 & 1 \\
160233 & 9.102 & 0.025 & 0.032 & 0.071 & $\mathrm{~B} 5$ & 3 & 0 & 2 \\
170783 & 7.717 & 0.187 & 0.016 & 0.593 & $\mathrm{~B} 5$ & 4 & 1 & 2 \\
171491 & 7.952 & 0.541 & -0.056 & 0.201 & $\mathrm{~B} 5$ & 1 & 0 & 2 \\
175544 & 7.395 & 0.144 & 0.002 & 0.171 & $\mathrm{~B} 3$ & 1 & 1 & 2 \\
177549 & 9.257 & 0.300 & -0.092 & 0.325 & $\mathrm{~B} 3$ & 1 & 0 & 2 \\
180126 & 8.012 & 0.207 & -0.029 & 0.361 & $\mathrm{~B} 3$ & 1 & 0 & 2 \\
\hline \hline
\end{tabular}

Ref: 1. Olsen 1983, 2. Knude 1992, 3. Schuster \& Nissen 1989.
TABLE 3. Numbers of measured stars.

\begin{tabular}{lccccc}
\hline \hline Bands & Bas 1 & Ber 86 & NGC 6704 & NGC 6756 & Total \\
\hline$y, b$ & 604 & 160 & 533 & 368 & 1665 \\
$u, v, b, y$ & 121 & $59^{\mathrm{a}}$ & 165 & 139 & 484 \\
\hline \hline
\end{tabular}

${ }^{a}$ The 5 brightest stars in Ber 86 are saturated in the $u$-frame.

the same field with respect to the brightest frame in the $y$ band. The short and long frames for Berkeley 86 were reduced separately, and the final instrumental magnitudes in each band were obtained from weighted averages of both frames.

The IRAF/DAOPHOT package (update 1994 January) was used to obtain aperture photometry, and PSF fitting and aperture corrections for the problem stars. The errors of the aperture correction, defined as the rms deviation of the corrections for about 10 stars per frame, are between 0.01 and 0.02 for the $1.5 \mathrm{~m}$ telescope frames, and 0.05 for the 1.23 telescope frames. In Table 3 we present the number of stars measured in at least two bands $(y, b)$ and in the four $u v b y$ bands, in the respective cluster fields.

Atmospheric extinction corrections were performed following a multinight method (see Young 1974), whereby extinction coefficients are calculated every night, as the mean of the coefficients for all the standard stars measured. The errors of the coefficients (rms deviation) amount to 0.01, whereas those of the extinction corrected magnitudes are most frequently below 0.01 . With the extinction corrected magnitudes, the following set of transformations are obtained for each telescope:

1.5 telescope:

$y_{i}=4.175+1.036 V-0.074(b-y) \quad 0.03$

$(b-y)_{i}=0.138+1001(b-y) \quad 0.02$

$c_{1, i}=-0.458+1.018 c_{1}-0.185(b-y) \quad 0.05$

$(u-b)_{i}=0.569+1.024(u-b)+0.171(b-y) \quad 0.03$

1.23 m telescope:

$y_{i}=6.100+1.027 V-0.108(b-y) \quad 0.04$

$(b-y)_{i}=0.180+1.107(b-y) \quad 0.01$

$c_{1, i}=-0.473+0.577 c_{1}-0.316(b-y) \quad 0.02$

$(u-b)_{i}=0.084+0.814(u-b)+0.259(b-y) \quad 0.06$

$m_{1}=\left[(u-b)-2(b-y)-c_{1}\right] / 2$,

where the subindex $i$ stands for instrumental magnitudes. The corresponding standard errors, defined as the rms deviation of the residuals, are also quoted.

Concerning the $V$ transformation, it has been pointed out that a coefficient of the $y$ magnitude slightly but significantly smaller than 1 might be a feature of instrumental systems with a CCD (Jønch-Sørensen \& Knude 1990; JønchSørensen 1993). This possibility is tested for our both instrumental systems. As to the $m_{1}$ index, it is computed from the transformed $(b-y), c_{1}$, and $(u-b)$ indices. This procedure is advisable to diminish as much as possible the systematic errors in the standard values caused by the unavoidable extrapolation of the transformations (Delgado \& Alfaro 1989). 


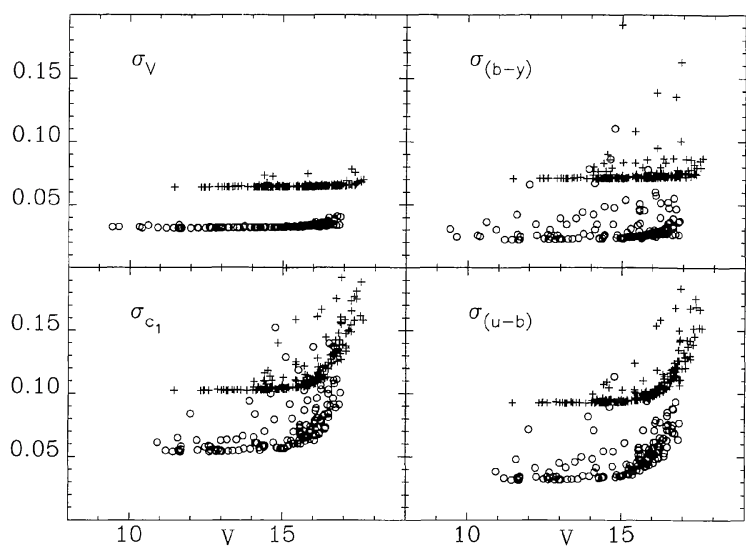

FIG. 2. Total estimated $\sigma$ 's for the standard $V,(b-y), c_{1},(u-b)$ plotted vs $V$. Crosses, $1.23 \mathrm{~m}$ telescope; circles, $1.5 \mathrm{~m}$ telescope. Only stars selected as members from their locations in at least two CM diagrams are plotted (Sec. $3.1)$.

An estimate of the total uncertainty affecting our standard indices can be gained from Fig. 2, where the total $\sigma$ 's (square root of the added squared errors mentioned above: PSF-fitting, aperture correction, extinction, and transformation) are plotted versus $V$, for $V,(b-y), c_{1},(u-b)$. These plots show only those stars considered cluster members from the location of their representative points in at least two color-magnitude (CM) diagrams (see Sec. 3.1 below). For the selected B-type members, which are the ones used to compute the color excess (see also Sec. 3), the largest errors in $V,(b-y), c_{1},(u-b)$ amount to $0.06,0.08,0.12,0.11$, and $0.03,0.04,0.07,0.05$ for the 1.23 and 1.5 observations respectively. A table with the complete photometry for the 1665 stars measured at least in $y$ and $b$ bands, together with coordinates $X, Y$ in the fields sketched in Fig. 1, can be obtained from the authors on request.

\section{PHOTOMETRIC DIAGRAMS}

\subsection{Member Stars and Reddening Slopes}

The first step in the analysis is the estimate of membership for the stars measured in each field. This can be done with the use of several CM diagrams, and the requirement that the stars lie close to the main sequence concentration in all them. Here we use the three CM diagrams $V-(b-y)$, $V-(u-b)$, and $V-(v-y)$. Stars selected as members have to lie at distances not larger than 0.2 from eye-estimated lines along the main sequence concentration in each diagram. In Fig. 3 we show as example the $\mathrm{CM}$ diagrams $V-(b-y)$ for the four clusters, with these lines drawn. The distance chosen for selection takes into account not only the errors in $V$ and the different color indices, but also the expected presence of variations in color excess, which can reach several tenths of a magnitude. In addition, we select as members the stars lying in similar positions in the giant region in all diagrams. Obviously, the only criterion available here to decide the membership of giant stars to the clusters is based on their photometric colors. The location of these stars in the diagrams for the three older clusters is however a

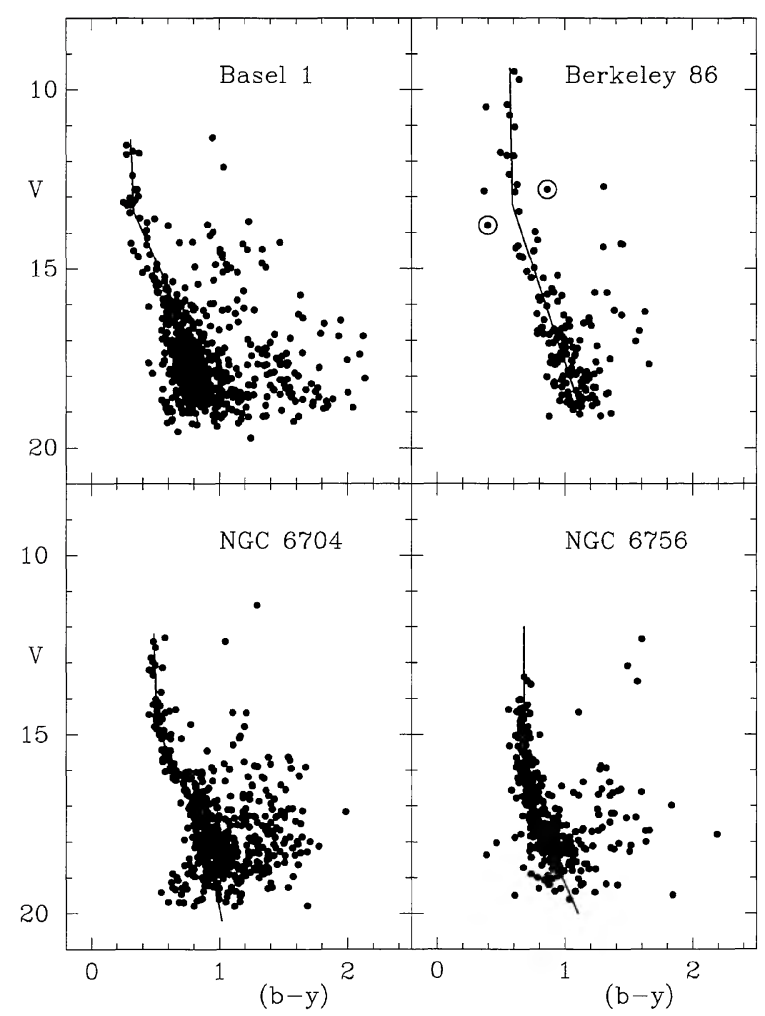

FIG. 3. $V-(b-y)$ diagram for the four clusters. The lines used for member selection are plotted as example. For comments on the two circled dots in Berkeley 86 (Sec. 3.1)

strong indication in favor of their membership. As we shall see later, the three clusters Basel 1, NGC 6704, and NGC 6756 have ages similar to the Hyades, and the presence of giant members should be expected.

The first members selection from this procedure, is now refined with the use of the diagrams $c_{1}-(b-y)$, $(u-b)-(b-y)$, and $c_{1}-(u-b)$. These diagrams help also in establishing an spectral classification between stars earlier and later than spectral type $A 0$, and estimating of mean reddening slopes, appropriated to the particular region. For these estimates, we use the reference lines for unreddened stars given by Crawford (1978, 1979, and 1975, for B-, A-, and F- type stars, respectively). The reference line for intermediate A-type stars by Hilditch et al. (1983), linking the B and A ranges of the Crawford's lines is also used.

Figure 4 shows as example the mentioned two color diagrams for the cluster Basel 1, with reference lines shifted according to the quoted values of color excess, and reddening slopes. Circled dots indicate the stars selected as B-type members, according to their locations in all three diagrams. The reddening slopes $\mathrm{E}\left(c_{1}\right) / \mathrm{E}(b-y), \mathrm{E}(u-b) / \mathrm{E}(b-y)$ estimated with this procedure are $0.20,1.60 ; 0.20,1.40 ; 0.30$, 1.50 for Basel 1, NGC 6704, and NGC 6756. For Berkeley 86 the available A- and F- type stars are very scarce. Here we are helped by published spectral types for some cluster members (Forbes et al. 1992, hereafter F92). Unfortunately, the five brightest stars in our field are saturated in the $u$ frame. Only for star No. 6 in the photometry of F92 we have 

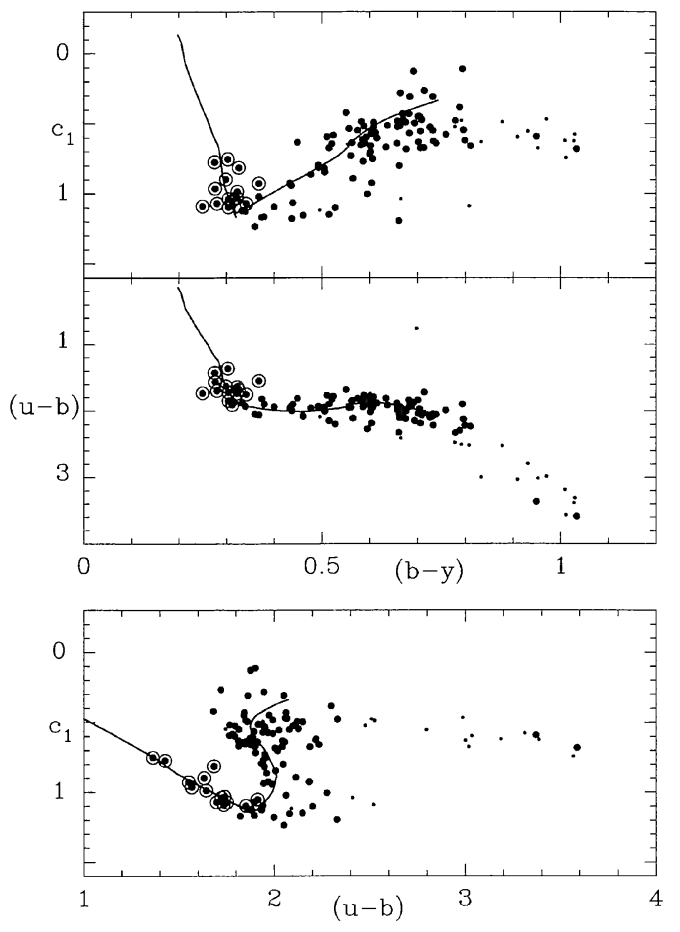

FIG. 4. Two color diagrams for Basel 1. The reference lines are shifted with the values: $\mathrm{E}(b-y)=0.33, \mathrm{E}\left(c_{1}\right) / \mathrm{E}(b-y)=0.20, \mathrm{E}(u-b) / \mathrm{E}(b-y)=1.60$. The circled dots are the selected B-type members.

spectral type (B2 V) and measured $(u-b)$ and $c_{1}$ indices. The slopes used, $0.18,1.52$, have been computed for this star, using the calibration of intrinsic colors in terms of spectral type given by Warren (1976). Furthermore, two of the stars given as members by F92 (Nos. 24, 26), first excluded by our criterion, have also been included as B-type members in Berkeley 86. They are indicated with circled dots in Fig. 3. In all cases, the slope for the $m_{1}$ index is computed as $\mathrm{E}\left(m_{1}\right)$ / $\mathrm{E}(b-y)=\left[\mathrm{E}(u-b) / \mathrm{E}(b-y)-2-\mathrm{E}\left(c_{1}\right) / \mathrm{E}(b-y)\right] / 2$.

A quantitative estimate of the uncertainties of these slopes is difficult to obtain, since they are based on visual fits. However, they will be obviously affected by at least the same errors than those of the corresponding photometric indices of the selected B-type members (see Sec. 2). In view of these errors, the differences of the slopes used here with those obtained by Crawford \& Mandwewala (1976: $\mathrm{E}\left(c_{1}\right)$ / $\mathrm{E}(b-y)=0.20, \mathrm{E}(u-b) / \mathrm{E}(b-y)=1.54)$ cannot be considered significant.

\subsection{Photometric Parameters}

3.2.1 Reddening and metallicity

Once the reddening slopes are estimated, we proceed to determine the intrinsic colors for individual stars. We can do this only for stars earlier than $\mathrm{A} 0$, as the calibration of $(b-y)_{0}$ for later stars depends on the $\mathrm{H} \beta$ index. The stars selected as B-type members are dereddened with the formulas given by Shobbrook (1984). For the remaining stars selected as members, the color excess is computed as the mean of the values obtained for individual B-type members. The values of color excess obtained amount to
$0.33 \pm 0.04,0.71 \pm 0.10,0.54 \pm 0.03,0.73 \pm 0.04$ for Basel 1, Berkeley 86, NGC 6704, and NGC 6756, respectively. Otherwise, the intrinsic largest error of the color excess for one star can be estimated from the photometric errors given in Sec. 2 (see Phelps \& Janes 1994, for an explanation of the quantitative estimates). They are 0.07 and 0.13 for the 1.5 (Basel 1, Berkeley 86) and 1.23 (NGC 6704, NGC 6756) measurements, respectively, which are to be compared with the rms deviations of the mean, given above. Therefore, no signs of differential or variable reddening above the photometric errors can be guessed from our data, with the exception of Berkeley 86.

An estimate of the metallicity could be attempted, at least for Basel 1 and NGC 6704, where we have a larger number of observed F-type stars. The computation with the available calibrations of $[\mathrm{Fe} / \mathrm{H}]$ in terms of $\delta m_{0}(b-y)=m_{0, \text { line }}$ $-m_{0 * *}$ (Crawford 1975; Crawford \& Perry 1976), produces the mean $[\mathrm{Fe} / \mathrm{H}]$ values $-0.3 \pm 0.7,0.6 \pm 1.3$ for Basel 1 and NGC 6704, respectively. The large uncertainties reflect the large errors of our photometric indices for the weakest members. Measurements of $\mathrm{H} \beta$, and longer integration times also in the $v$ and $u$ bands, should improve this determination through a determination of individual reddening for AF stars, use of $\delta m_{0}(\beta)$, more precise membership determination, and, obviously, more precise values for the magnitudes in the different bands.

\subsubsection{Distances and ages}

As has been also exemplified by Turner (1994), local variations of the reddening slopes are in some cases associated with variations of $R$, the ratio of absorption in $V$ to color excess $\mathrm{E}(B-V)$. How these variations might behave in the Strömgren system is unknown. On the other hand, the reddening slopes used here are not significantly different from the average values commonly used (Crawford \& Mandwewala 1976). We assume $R=3$, and compute the absorption coefficient for each cluster with the relation between $\mathrm{E}(B-V)$ and $\mathrm{E}(b-y)$ given by Penprase (1992). Distance moduli are then obtained by shifting the observational main sequence to fit the dereddened $\mathrm{CM}$ diagram $V_{0}-(b-y)_{0}$. The results are $11.0 \pm 0.3,11.1 \pm 0.3,11.3 \pm 0.4,12.6 \pm 0.4$ for Basel 1, Berkeley 86, NGC 6704, and NGC 6756, respectively. The uncertainties of these values have been estimated from the photometric errors, following the reasoning by Phelps \& Janes (1994).

To estimate the ages we dispose of recent model isochrones, provided for specific ages by Claret (1996, private). All the isochrones used here are for solar chemical composition $(Z=0.02, X=0.7)$. The models include the consideration of newest opacities and energy generation rates. A moderate amount of overshooting from the convective core is parametrically included in the computations (see Claret 1995, and F. Pérez-Hernández et al. 1995, for a description of the models, and the calibrations used of temperature and bolometric correction in terms of $U B V$ colors). The isochrones are translated to the Strömgren color-magnitude diagrams, $V_{0}-(b-y)_{0}$, and $V_{0}-(u-b)_{0}$, with the well calibrated formulas given by Penprase (1992) for $(b-y)_{0}$ and Turner $(1990)$ for $(u-b)_{0}$. In Fig. 5 we show the intrinsic 

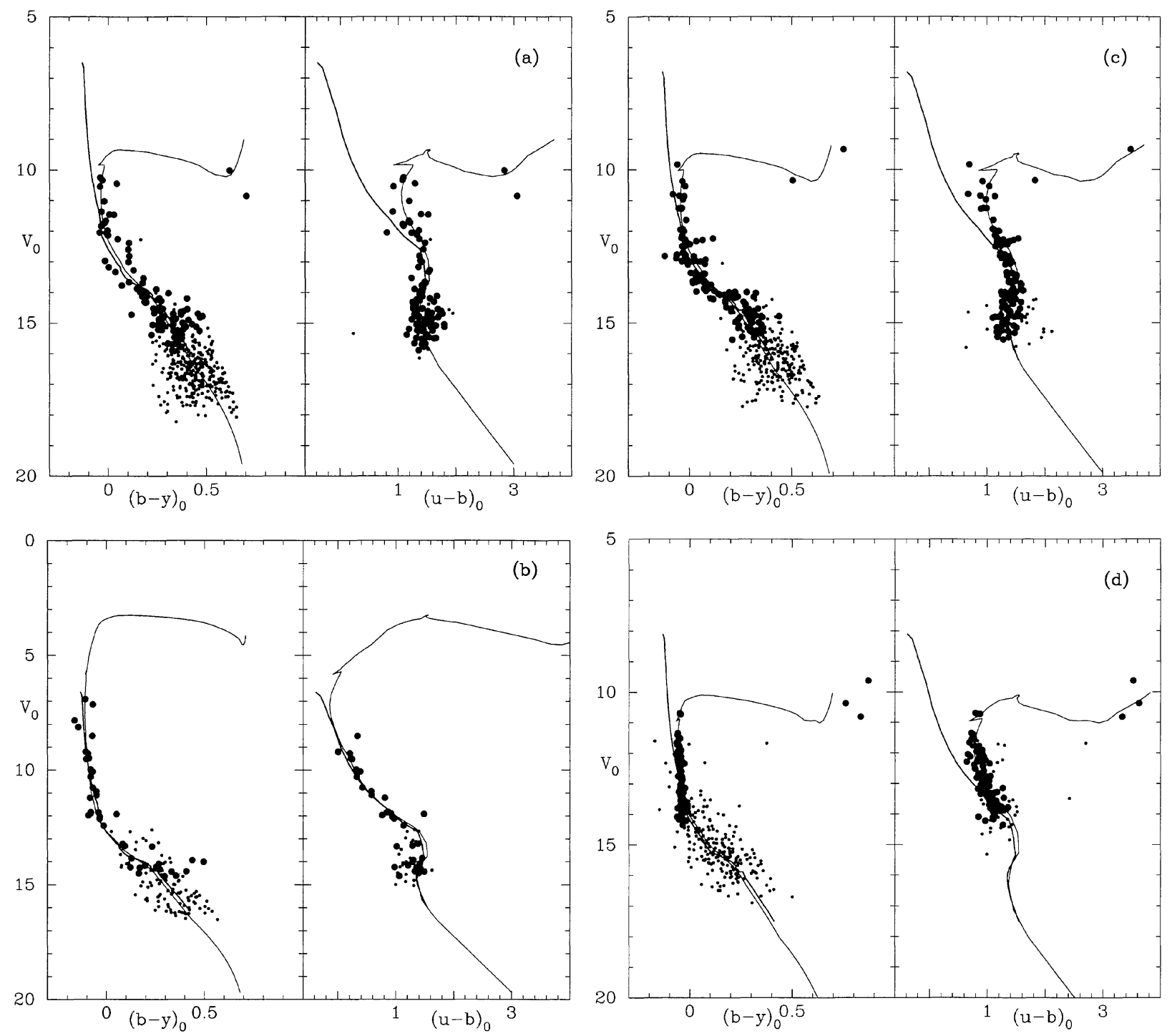

FIG. 5. (a)-(d). Isochrone fits to the dereddened $V-(b-y)_{0}$, and $V-(u-b)_{0}$ diagrams: (a) Basel 1, (b) Berkeley 86, (c) NGC 6704, (d) NGC 6756. The corresponding ages and distance moduli are listed in Table 4. Empirical ZAMS lines are plotted as thicker lines. Larger dots indicate members in all three CM diagrams, according to our criterion (Sec. 3.1).

color magnitude diagrams of the four clusters, with the best isochrone fits superimposed. The errors of these ageestimates can only be guessed qualitatively, on the basis of the errors of the photometric points, and the variations of the fit quality with varying age of the isochrone, in both $\mathrm{CM}$ diagrams simultaneously. We estimate ages of $2.3 \pm 0.3 \times 10^{8} \mathrm{yr}, 8 \pm 5 \times 10^{6} \mathrm{yr}, 2.0 \pm 0.6 \times 10^{8} \mathrm{yr}, 1.3 \pm 0.6$ $\times 10^{8} \mathrm{yr}$, for Basel 1 , Berkeley 86 , NGC 6704, NGC 6756, respectively. The best guesses, based on these fits, are included in Table 4, together with our estimates for the distance moduli and color excess, compared to values given in the literature.

We wish to stress here the adequacy of this isochrone calibration, via color transformation, that can be appreciated in the good agreement between the transformed isochrones, and the observational $u v b y$ main sequence lines, also plotted in Fig. 5 as thicker continuous lines. Furthermore, we con- sider our age estimates as reliable, in view of the satisfactory simultaneous fits of theoretical intrinsic colors, from two independent calibrations, to our two CM diagrams, in a relatively large range of spectral types along the main sequence.

\subsection{Comparison with Previous Studies}

Photometric studies have been published for the four clusters in the present report. The amount of information is variable, but all them have at least one determination of the basic photometric parameters, color excess, age, and distance. Otherwise, none of them has published $u v b y$ photometry, and only some stars in Berkeley 86 have published spectral types (Mermilliod, Garrison: private). The three clusters Basel 1, NGC 6704, and NGC 6756 were measured in the photographic $R G U$ system some decades ago (Fenkart 1965; Grubbisich 1965; Slovopoulos 1965). Except for NGC 6704, 
TABLE 4. Clusters parameters compared to published estimates.

\begin{tabular}{|c|c|c|c|c|}
\hline Cluster & $E(b-y)$ & $\begin{array}{c}\text { Log Age } \\
\text { (years) }\end{array}$ & $D M_{0}$ & $\mathrm{E}(B-V)$ \\
\hline Bas 1 & 0.33 & $\begin{array}{l}8.35 \\
7.76(0)\end{array}$ & $\begin{array}{l}11.00 \\
10.67(0) \\
11.00(1) \\
10.62(2) \\
11.02(3)\end{array}$ & $\begin{array}{l}0.43 \\
0.57(0)\end{array}$ \\
\hline Ber 86 & 0.71 & $\begin{array}{l}6.94 \\
6.78(0) \\
6.75(4) \\
6.78(5) \\
6.40(6) \\
6.70(7)\end{array}$ & $\begin{array}{l}11.10 \\
10.23(0) \\
11.18(5) \\
11.39(6) \\
11.01(7)\end{array}$ & $\begin{array}{l}0.89 \\
0.99(0) \\
0.96(5) \\
0.80(6) \\
1.01(7)\end{array}$ \\
\hline NGC 6704 & 0.54 & $\begin{array}{l}8.30 \\
7.30(0)\end{array}$ & $\begin{array}{l}11.30 \\
11.44(0) \\
11.29(2) \\
11.40(8)\end{array}$ & $\begin{array}{l}0.69 \\
0.72(0) \\
0.71(8)\end{array}$ \\
\hline NGC 6756 & 0.73 & $\begin{array}{l}8.12 \\
7.67(0) \\
8.48(9)\end{array}$ & $\begin{array}{l}12.60 \\
10.89(0) \\
11.09(10)\end{array}$ & $\begin{array}{l}0.91 \\
1.18(0)\end{array}$ \\
\hline
\end{tabular}

REFERENCES.-(0) Lyngå, Open Clusters Catalog. (1) Becker \& Fenkart 1970. (2) Grubissich 1965. (3) Fenkart 1965. (4)Smith et al. 1988. (5) Forbes 1981. (6) Massey et al. 1995. (7) Forbes et al. 1992. (8) Forbes \& DuPuy 1978. (9) Santos et al. 1993. (10) Slovopoulos 1965.

for which $U B V$ photometry has been published more recently (Forbes \& DuPuy 1978, hereafter referred to as F78), these $R G U$ works contain the only available estimates for the cluster parameters. For Berkeley 86, $U B V$ photometry has been published by Forbes (1981), F92, and Massey et al. (1995).

\subsubsection{Comparison of individual stars}

The comparison with previously published photometric indices can be performed for the clusters Berkeley 86 and NGC 6704, after transformation of our $u v b y$ indices $(b-y)$ and $(u-b)$ with formulas given by Turner (1990). The average differences of the stars in common with the $U B V$ photometric study by F92, in the sense F92-ours, are $\Delta V=0.10 \pm 0.15, \Delta(B-V)=0.01 \pm 0.03, \Delta(U-B)=-0.00$ \pm 0.07 .

The agreement is quite satisfactory, but a systematic difference appears in the $V$ values, whereas the differences in $(B-V)$ and $(U-B)$ are smaller and not systematic. The difference in $V$ is significantly decreased using the classical transformation to obtain the $V$ standard magnitudes (Crawford \& Barnes 1970; see Sec. 2 above):

$$
y_{i}-V=4.478-0.117(b-y) \quad \text { St.error }=0.03
$$

which gives a difference $\Delta V=-0.06 \pm 0.13$. Figure 6 shows the two sets of magnitudes compared to those in F92. Two of the stars in common, (stars 3, 4 in F92), denoted with squares in the figure, still show large differences with our values. On the other hand, the mean difference with the CCD values obtained by Massey et al. (1995) for three stars in common $(1,2$, and 4 in F92) are much smaller, $\Delta V=-0.009 \pm 0.009$ in the sense Massey-ours. We note that

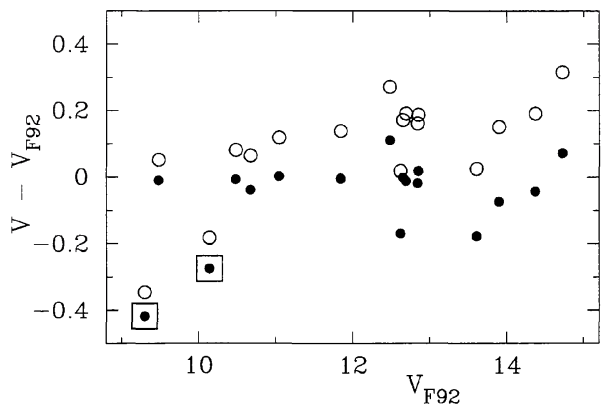

FIG. 6. Differences between our $V$ magnitudes and those in F92, for the stars in common in Berkeley 86 . The results of two transformations to the standard system are shown. Circles: $V$ transformation with variable $y$ coefficient. Dots: $V$ transformation with $y$ coefficient equal to 1 . For comments on the two squared dots (Sec. 3.3.1).

stars F92-3 and F92-4 are double, and probably very hardly separable in photoelectric photometry. Rejecting them, the average difference F92-ours reduces to $\Delta V=-0.02 \pm 0.07$. On the basis of the good agreement with the photoelectric values in F92, and those of Massey et al. as well, we use the transformation equation with the $y$-coefficient equal to 1 for the measurements in the $1.5 \mathrm{~m}$ telescope instrumental system.

For NGC 6704, we compare with the results in F78, the only $U B V$ photometric study known to us. The information contained in the tables of F78 is somewhat confusing, making uncertain the comparison with their photographic values. Of the 41 stars in common with us, 5 have photoelectric measurements in F78 (stars A, B, F, H, J, whereas A, F, and $\mathrm{H}$, also have photographic measurements). Restricting the comparison to these 5 stars, we obtain mean values, $\Delta V=-0.01 \pm 0.07, \quad \Delta(B-V)=-0.01 \pm 0.27, \quad \Delta(U-B)$ $=0.07 \pm 0.27$. The differences are not very big, but the dispersions are too high, well above the errors of either our photometry, or the one in F78. We cannot think of a reasonable explanation for that. We just note that the mean differences in F78, between their own photographic and photoelectric values for the three stars with both kind of measurements, are already very high, $\Delta V=0.04 \pm 0.11$, $\Delta(B-V)=0.10 \pm 0.07, \Delta(U-B)=0.28 \pm 0.17$.

$$
\text { 3.3.2 Membership }
$$

Membership assignments are given in the photometric studies cited above for all four clusters, using the location of the representative points in the respective photometric diagrams. Some differences with our assignments are present, mainly for stars of spectral types around BA, and later. In general we keep our estimates, considering them more confident on the basis of the wider range of spectral types measured, and the disposal of 3 independent color magnitude diagrams.

We just comment on two differences here. First, stars 24, 26, in Berkeley 86 (F92 numbers), were first rejected by us because of their $(b-y)$ values, although their locations in the other CM diagrams were inside the distance chosen for selection. The stars are considered members by F92, based on their photoelectric colors, and we have indeed included 


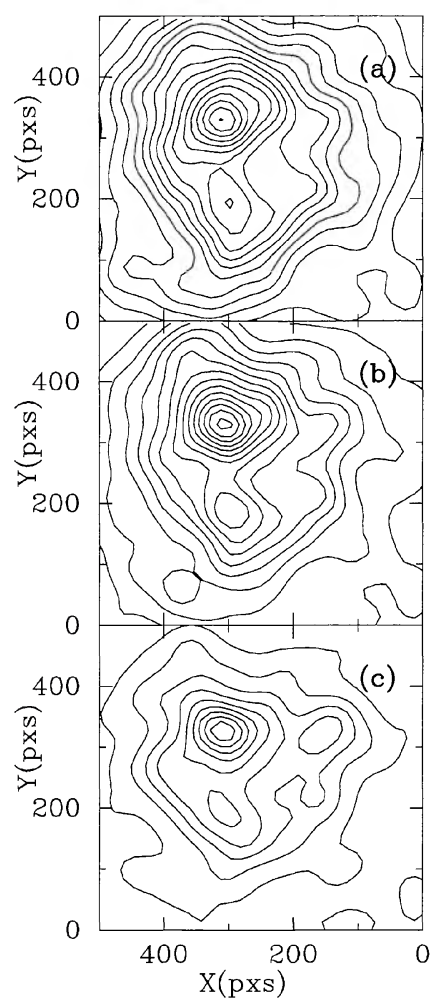

FIG. 7. Density contours of member stars in the field of NGC 6756 shown in Fig. 1(d). (a) All members. (b) $V \leqslant 18$. (c) $V \leqslant 16.5$.

them as B-type members in the computation of the color excess for the cluster. Second, in none of the published studies on the clusters Basel 1, NGC 6704, and NGC 6706 is there a mention to the membership of giant stars. In these three clusters, we suggest some giant stars as members, on the basis of their location in all CM diagrams. In Fig. 5, showing the dereddened CM diagrams, we observe the acceptable location of these stars, in spite of the rough estimates of their color excesses. As has been commented above, with the ages obtained for these clusters, they are expected to have an incipient red giant branch.

The spatial distribution of members has been only analyzed for NGC 6756, the farthest cluster in our sample, where the observed field is expected to comprise more completely the cluster dimensions. Some signs of mass segregation can be observed in that case. In Fig. 7 we show density contours, for member stars in three $V$ magnitude ranges: All stars, $V \leqslant 18$, and $V \leqslant 16.5$. This plots reveal stronger concentration for brighter members.

\subsubsection{Comparison with published ages and distances}

In Table 4 we have also included the available published values for the cluster parameters. As can be seen, our estimates for the ages are very similar, and larger than $10^{8}$ years for all three clusters Basel 1, NGC 6704, and NGC 6756. In the catalog of Lyngå (reference 0 in Table 4), these objects have quoted ages sensibly below this figure, and in particular for NGC 6756, the existing estimates for distance are sensibly different from our results, the difference being above the errors estimated for our photometry. We consider our values more realistic, in view of the acceptable fits to both observational reference lines, and isochrones, for a wide range along the main sequence. The three clusters seem in fact to be the same object, just observed at different distances. In particular, we point out the improvement of the age estimate for NGC 6704, which turns out to be significantly older than previously estimated.

Berkeley 86 is a much younger object. The age obtained here is in good agreement with previously published values, but our absolute distance modulus is smaller than other recent determinations by 0.1 to 0.3 . We have seen in the previous subsection that the agreement with the values of reddening, and of color indices for the stars in common, in particular in $V$, is acceptable. On the other hand, the value of distance modulus obtained by Massey et al. (1995) is larger than ours by 0.3 , and is based on spectral types. This difference is most probably due to the region covered by the observations of Massey et al., of about half a degree, while our observations are restricted to the more reddened cluster core. In fact, they obtain a lower color excess, $\mathrm{E}(B-V)=0.8$, compared to our value $\mathrm{E}(b-y)=0.71$ which results in $\mathrm{E}(B-V)=0.89$ with the relation by Penprase (1992). With a value for the absorption coefficient $R=3$, also used in their work, this causes a difference in distance modulus of 0.3 , which accounts for the discrepancy.

\section{SUMMARY AND CONCLUSIONS}

We have obtained improved measurements of color excess, distance and age for four galactic clusters. The new estimates differ from previously published values in some issues. We point out the ages obtained for the three clusters Basel 1, NGC 6704, and NGC 6756, which turn out to be older than previously assumed, in particular for the two first ones. As to NGC 6756, its distance is distinctly larger than the values estimated by other authors.

We indeed consider our results as an improvement, as they are based on fits to both reference lines, and updated model isochrones on a wide range of spectral types from $B$ to F.

The membership of giant stars is suggested in the three clusters Basel 1, NGC 6704, and NGC 6756, from their locations in three color magnitude diagrams. In this last cluster, signs of mass segregation are detected, in the sense of the most massive members being more concentrated towards the cluster core.

We are grateful to Eloy Rodríguez, for allowing us the use of the observing time granted to him in the $1.23 \mathrm{~m}$ telescope at the CAHA. Private communications by R. F. Garrison, on spectral types, and J. C. Mermilliod, who provided us with his Open Clusters Data Base, are acknowledged. This work has been financially supported by the spanish DGICYT, through grants numbers PB91-0053, PB94-0007, and by the Research and Education Council of the Autonomous Government of Andalucía (Spain). The observations have been taken at the German-Spanish Observatory of Calar Alto (Spain). 


\section{REFERENCES}

Alfaro, E. J., \& Delgado, A. J. 1991, A\&A, 241, 69

Becker, W., \& Fenkart, R. 1971, A\&AS, 4, 241

Claret, A. 1995, A\&AS, 109, 441

Crawford, D. L. 1975, AJ, 80, 955

Crawford, D. L. 1978, AJ, 83, 48

Crawford, D. L. 1979, AJ, 84, 1858

Crawford, D. L., \& Barnes, J. V. 1970, AJ, 75, 978

Crawford, D. L., \& Mandwewala, N. 1976, PASP, 88, 917

Crawford, D. L., \& Perry, 1976, PASP, 88, 454

Delgado, A. J., \& Alfaro, E. J. 1989, A\&A, 219, 121

Delgado, A. J., Alfaro, E. J., García Pelayo, J. M., \& Garrido, R. 1992, AJ, 103,891

Fenkart, R. P. 1965, ZfA, 62, 90

Forbes, D. 1981, PASP, 93, 441

Forbes, D., \& DuPuy, D. L. 1978, AJ, 83, 266 (F78)

Forbes, D., English, D., DeRobertis, M. M., \& Dawson, P. C. 1992, AJ, 103, 916 (F92)

Grubissich, Cl. 1965, ZfA, 60, 249

Hilditch, R. W., Hill, G., \& Barnes, J. V. 1983, MNRAS, 204, 241
Jønch-Sørensen, H. 1993, A\&A, 267, 54

Jønch-Sørensen, H., \& Knude, J. 1990, A\&A, 238, 75

Knude, J. 1992, A\&AS, 92, 841

Lyngå, G. 1987, Catalogue of Open Cluster Data (Lund Observatory)

Manfroid, J., \& Sterken, C. 1987, A\&AS, 71, 539

Massey, P., Johnson, K. E., \& DeGioia-Eastwood, K. 1995, ApJ, 454, 151

Olsen, E. H. 1983, A\&AS, 54, 55

Penprase, B. E. 1992, ApJS, 83, 273

Pérez-Hernández, F., Claret, A., \& Belmonte, J. A. 1995, A\&A, 295, 113

Phelps, R. L., \& Janes, K. A. 1994, ApJS, 90, 31

Santos, J. F. C., \& Bica, E. 1993, MNRAS, 260, 915

Schuster, W. J., \& Nissen, P. E. 1988, A\&AS, 73, 225

Shobbrook, R. R. 1984, MNRAS, 211, 659

Slovopoulos, S 1965, ZfA, 61, 105

Smith, L. F., Meynet, G., \& Mermilliod, J. C. 1988, A\&A, 287, 835

Turner, D. G. 1990, PASP, 102, 1331

Turner, D. G. 1994, RMxA\&A, 29, 163

Warren, W. H. 1976, MNRAS, 174, 111

Young, A. T. 1974, Methods Exp. Phys., 12A, 123 